Results Mean overall serum dioxin level was: 31.4 pg CALUX TEQ/g fat (mean values for the general Flemish population: 35.0-41.8 pg). Maintenance workers had significantly $(\mathrm{p}<0.05)$ lower serum dioxin levels (mean $26.9 \mathrm{pg})$ than garage workers $(38.3 \mathrm{pg})$ and administrative workers $(38.8 \mathrm{pg})$. Maintenance workers with $>10$ years seniority had lower serum dioxin levels $(24.7 \mathrm{pg})$ than those with $\leq 10$ years seniority $(31.5 \mathrm{pg})$. This difference was not significant.No higher serum dioxin concentrations were found in smokers, high BMI, living near a road with heavy traffic.

Conclusion The results showed no increased dioxin-like serum levels in maintenance workers in the two municipal waste incinerators. These results seems to indicate that there is no additional risk for dioxin exposure in the two considered waste incinerators.

\section{OCCUPATIONAL EXPOSURE TO SELENIUM COMPOUNDS, ITS EFFECT ON BIOLOGICAL PARAMETERS AND MARKERS OF DIABETES}

A Greiner* ${ }^{*}$ R Feltes, J Hildebrand, T Göen, H Drexler. Institute of Occupational, Social and Environmental Medicine, Univ. of Erlangen-Nürnberg, Erlangen, Germany

\subsection{6/oemed-2018-ICOHabstracts.1147}

Introduction Selenium (Se) exhibits a very small safety margin between recommended daily intake and harmful dosages (e.g. elevated risk for diabetes). Volunteer exposure studies have indicated different metabolisms for organic and inorganic Se compounds. The objective of this study was to establish a comprehensive biological monitoring approach for elemental/ inorganic Se exposure at workplaces and to evaluate its potential effects in men.

Methods In a case-control study we determined total Se in plasma and urine, Se speciation in urine, Glutathione peroxidase activity, prothrombin time, glucose, HbA1c and proinsulin in 18 employees of a Se processing plant and 18 age matched controls without occupational Se exposure. For the Se-exposed group we additionally measured the average shift level of total Se and of water-soluble Se-fraction in air, Se in urine at the beginning and at the end of the shift, and after at least two weeks off-work.

Results Se plasma levels of the exposed individuals ranged between 62 and $123 \mu \mathrm{g} / \mathrm{L}$ and urine levels after shift ranged between 22 and $340 \mu \mathrm{g} / \mathrm{g}$ creatinine. Both were significantly higher than the levels in the controls. The air exposure to total Se ranged between $<\mathrm{LOD}$ and $2394 \mu \mathrm{g} / \mathrm{m}^{3}$. For glutathione peroxidase activity, prothrombin time, glucose, HbA1c and proinsulin there were no significant differences between the two groups.

Conclusion At workplaces in the selenium processing industry considerably high air concentrations of Se were detected, which exceeded the German exposure limit (MAK $20 \mu \mathrm{g} / \mathrm{m}^{3}$ ). In contrast, the biological tolerance limit (BAT $150 \mu \mathrm{g} \mathrm{Se} / \mathrm{L}$ plasma) was not exceeded. These diverging results necessitate a differentiated look on Se at workplaces. The workplace conditions including the kind of selenium (elemental, inorganic, water-soluble, organic) should be considered. A revision of selenium threshold values seems advisable.

\section{URINARY BLADDER CANCER IN A FORMER AREA OF} COAL, IRON AND STEEL INDUSTRIES IN GERMANY

${ }^{1} \mathrm{~K}$ Golka*${ }^{2,3} \mathrm{D}$ Ovsiannikov, ${ }^{4,5} \mathrm{E}$ Krech, ${ }^{1} \mathrm{M}$ Blaszkewicz, ${ }^{3} \mathrm{O}$ Moormann, ${ }^{5} \mathrm{MC}$ Truss, ${ }^{6}$ MW Haenel, 'JG Hengstler, 'S Selinski. 'Leibniz Research Centre for Working Environment and Human Factors (IfADo), Dortmund, Germany; ${ }^{2}$ Department of Urology and Paediatric Urology, Kemperhof Hospital, Germany; ${ }^{3}$ Department of Urology, Josefs-Hospital DortmundHoerde, Dortmund, Germany; ${ }^{4}$ Department of Urology, Klinikum Lüdenscheid, Lüdenscheid, Germany; ${ }^{5}$ Department of Urology, Klinikum Dortmund, Dortmund, Germany; ${ }^{6}$ Max-PlanckInstitut für Kohlenforschung, Muelheim an der Ruhr, Germany

\subsection{6/oemed-2018-ICOHabstracts.1148}

Introduction Urinary bladder cancer risk in coal miners is currently under debate. We report on a recently performed study in 2 departments of urology in Dortmund, a centre of the former underground hard coal mining industry in Germany. In the greater Dortmund area in the 1990s when coal, iron and steel industries were still active, $70 \%$ of bladder cancer cases were glutathione S-transferase M1 (GSTM1) negative, compared to $54 \%$ in unexposed controls.

Methods A total of 400 bladder cancer cases and 442 controls with benign urological diseases but without a history of malignancies, were assessed by questionnaire from July 2009 to July 2013. Furthermore, all patients were genotyped for polymorphic enzymes relevant for bladder cancer like glutathione S-transferase M1 (GSTM1) and N-acetyltransferase 2 (NAT2). Results More than ten years after closure of these industries, the frequency of the GSTM1 negative genotype decreased to $52 \%$, which is a normal finding in general population (OR 0.96, 95\% CI: 0.73 to 1.26 ; smokers: OR 0.93 , 95\% CI: 0.66 to 1.30 ; non-smokers: OR $1.02,95 \% \mathrm{CI}: 0.58$ to 1.80 ). However, former hard coal miners had still an elevated bladder cancer risk (hospital A: 20 bladder cancer cases (10\%) and 8 controls (3\%) (OR 3.22, 95\% CI: 1.39 to 7.49); hospital B: 32 cases (16\%) and 20 controls (10\%) (OR 1.72, 95\% CI: 0.95 to 3.12 ). The slow $\mathrm{N}$-acetyltransferase 2 status, which is associated with an increased bladder cancer risk in persons formerly exposed to aromatic amines, was normal in hard coal miners with bladder cancer in all three studies in the Dortmund area.

Conclusion After closure of the coal, iron and industries in the 1990s, GSTM1 negative genotype is currently no more a relevant bladder cancer risk factor. However, an elevated bladder cancer risk in former hard coal miners is still observable.

\section{PROGNOSIS OF OCCUPATIONAL BLADDER CANCER AND POLYMORPHIC XENOBIOTIC METABOLISING ENZYMES}

${ }^{1} \mathrm{H}-\mathrm{M}$ Prager*, ${ }^{1} \mathrm{C}$ Lukas, ${ }^{2} \mathrm{M}$ Blaszkewicz, ${ }^{2} \mathrm{~T}$ Kadhum, ${ }^{2} \mathrm{JG}$ Hengstler, ${ }^{2} \mathrm{~S}$ Selinski, ${ }^{2} \mathrm{~K}$ Golka. ${ }^{1}$ Institute for Occupational, Social and Environmental Medicine, Castrop-Rauxel, Germany; 'Leibniz Research Centre for Working Environment and Human Factors at TU Dortmund (IfADo), Dortmund, Germany

\subsection{6/oemed-2018-ICOHabstracts. 1149}

Introduction In recent years, approximately 150 bladder cancer patients per year were acknowledged as an occupational disease in Germany. The question rises whether in genome-wide association studies described bladder risk factors may modulate the prognosis of occupational bladder cancer. 
Methods One hundred and thirty-six cancer patients surveyed for an occupational disease of the bladder were investigated for the course of the disease. EDTA blood samples were drawn. Patients were genotyped for the following polymorphisms: N-acetyltransferase 2 (NAT2, substrate: aromatic amines), glutathione S-transferase M1 (GSTM1, substrate: reactive metabolites of $\mathrm{PAH})$, glutathione S-transferase T1 (GSTT1, substrate: small molecules with 1 or 2 carbon atoms), UDP-glucuronyltransferase 1A2 rs11892031 (UGT1A2, substrate: aromatic amines), rs9642880 (close to c-Myc gene) and rs710521 (close to TP63). Frequencies of recurrences were analysed by means of chi-square test, relapse-free times were analysed by unadjusted Cox regression. The combined effect of the polymorphisms was analysed by means of the weighted polygenic risk score (PRS).

Results In $38 \%$ of the patients a recurrence was reported (median 1.54 years). All investigated polymorphisms except for rs710521 showed a tendency to more frequent recurrences and shorter recurrence-free times, in particular NAT2 (slow vs fast: hazard ratio $\mathrm{HR} 1.75,95 \% \mathrm{CI}: 0.98$ to 3.12 , $\mathrm{p}=0.0582$ ), GSTM1 (positive versus negative: HR 1.77, 95\% CI: 0.70 to $4.48, \mathrm{p}=0.2222$ ) and GSTM1 (negative vs positive, HR 1.37, 95\% CI: 0.76 to $2,45, \mathrm{p}=0.2972$ ). The PRS was significantly associated with shorter recurrence-free times (PRS >median vs PRS Smedian score: 18 vs 26 months, $\mathrm{HR}=1.93$, 95\% CI: 1.06 to $3.53 ; \mathrm{p}=0.0327)$, the risk of recurrence was also higher $(47 \%$ vs $31 \%, \mathrm{OR}=1.94,95 \% \mathrm{CI}$ : 0.93 to $4.06, \mathrm{p}=0.0757)$.

Conclusion Polymorphic xenobiotic metabolising enzymes may modulate the prognosis of occupational bladder cancer.

\section{CASE STUDY IN DATA ACCESS AND REANALYSIS: DIESEL ENGINE EXHAUST AND LUNG CANCER MORTALITY IN THE DIESEL EXHAUST IN MINERS STUDY (DEMS) COHORT USING ALTERNATIVE EXPOSURE ESTIMATES AND RADON ADJUSTMENT}

Roger 0 McClellan. Toxicology and Risk Analysis, Albuquerque, New Mexico, USA

\subsection{6/oemed-2018-ICOHabstracts. 1150}

Introduction The Diesel Exhaust in Miners Study (DEMS), conducted by National Institute of Occupational Safety and Health (NIOSH) and National Cancer Institute (NCI), included 12315 workers with 200 observed lung cancers from 8 U.S. non-metal mines ( 3 trona, 3 potash, 1 salt and 1 limestone). Retrospective cohort and case-control analyses by NIOSH and NCI scientists yielded a positive association between diesel exhaust exposure (DEE), represented by a respirable elemental carbon (REC) metric estimated retrospectively from carbon monoxide measurements, and lung cancer mortality. This finding was a major factor in the International Agency for Research on Cancer (IARC) classification of DEE as a human carcinogen.

Methods Our team was given access to the DEMS data and conducted analyses to first replicate the original analyses and then conduct extended re-analyses. Our re-analyses focused on

a. use of an alternative exposure metric developed using historical data on diesel equipment, engine horse power and ventilation rates without dependence on use of carbon monoxide as a surrogate for REC,

b. inclusion of radon as a covariate in statistical models, and

c. subgroup heterogeneity.
Results We found associations with cumulative REC and average REC intensity using the alternative REC estimates were generally attenuated compared with those found using the original DEMS REC estimate. Most findings were statistically nonsignificant, especially after control for radon exposure, which substantially weakened associations with the original and alternative REC estimates. No significant findings were detected among all miners who worked exclusively underground. However, associations were anomalously strong among limestone miners; no association with REC or radon was found among workers at the other seven mines.

Conclusions The large differences in results based on alternative exposure estimates, control for radon, and stratification by worker location or mine type highlight areas of uncertainty and the limited robustness of the DEMS data. These limitations must be considered in any extrapolation of the DEMS findings to other populations, and especially in using them for quantitative risk assessment. Moreover, the recently complete Advanced Collaborative Emissions Study (ACES) study sponsored by the Health Effects Institute and conducted at the Lovelace Respiratory Research Institute, Albuquerque, NM, indicated that chronic inhalation exposure of rats to low dilutions of exhaust from new technology diesel engines did not produce lung cancer. The results of both the DEMS and ACES findings will be reviewed to provide perspective for evaluating the cancer hazards of diesel-powered equipment, past, present and future.

\section{2a EVALUATING EXPOSURE TO DIESEL ENGINE EXHAUST}

JM Berg*. Centre for Toxicology and Environmental Health, LLC; Texas, USA

\subsection{6/oemed-2018-ICOHabstracts.1151}

Introduction Exposure to diesel engine exhaust continues to be a concern for employers who utilise either traditional (TDE) or new technology diesel (NTDE) exhaust-powered equipment. Such exposure may occur at elevated concentrations over a short period of time (acute) or at much lower concentrations on a daily basis over their working lifetime (chronic). Individuals in each of these exposure scenarios may present with different symptomology or health effects. In either case, accurately assessing an individuals exposure to diesel exhaust should be done in the context of appropriate toxicological endpoint of relevance (e.g., irritation, carcinogenicity, etc.).

Methods A review of the toxicological and industrial hygiene literature was conducted to identify historical trends in exposure sampling methodology and analytical surrogate chosen. Additional studies were reviewed which highlighted the important compositional distinctions between TDE and NTDE and also the reported symptomology which presented following acute exposures.

Results Historically, a number of analytical surrogates for diesel exhaust have been utilised to assess exposure including both particulate phase and gaseous compounds. While at the present time, sampling for elemental carbon or respirable elemental carbon (REC) is currently the most utilised surrogate, recent literature indicates that the elemental carbon fractions of TDE and NTDE may substantially differ. Furthermore, a number of studies noted that acute exposure to diesel exhaust may elicit transient irritant or neurophysiological effects 